\title{
Electron Transport through Double Quantum Dot System with Inter-Dot Coulomb Interaction
}

\author{
D. SzTEnKIEl AND R. ŚwirkOwiCZ
}

Faculty of Physics, Warsaw University of Technology

Koszykowa 75, 00-662 Warsaw, Poland

(Received December 27, 2006; revised version March 6, 2007)

\begin{abstract}
A theoretical approach to a problem of electron transport through double quantum dot systems based on non-equilibrium Green function formalism using equation of motion method is presented. $I-V$ characteristics and differential conductance are calculated and discussed in detail in the intermediate regime with tunneling rate between the quantum dots comparable to coupling constants with external electrodes. Effects of inter-dot Coulomb correlations are studied for various values of interaction parameter $U$. It is shown that the interaction influences transport properties in a pronounced way and apart from the simple Coulomb blockade additional effects can be obtained. When energy levels of two quantum dots are not aligned, the asymmetry in conductance characteristics is closely related to a voltage dependence of population numbers in both quantum dots. For a one bias polarization electrons are well localized in quantum dots in a low voltage region, whereas for the opposite one they are partly delocalized.
\end{abstract}

PACS numbers: 73.23.-b, 73.21.La, 73.23.Hk

\section{Introduction}

Electron transport across quantum dot (QD) structures has been the subject of active experimental and theoretical studies during the last years. In particular, the idea of using coupled quantum dots in quantum computing devices attracted a great interest and experimental techniques were developed to investigate and to control double quantum dot (DQD) systems [1-3]. Quantum dots connected in series as well as in parallel configuration were intensively investigated [4-6]. $I-V$ characteristics obtained experimentally for such structures reveal asymmetries with respect to bias reversal [7]. Negative differential conductance was also observed [8]. The asymmetry in experimental setup arises mainly due to differ- 
ences between QDs induced by impurities, unintentional charging effects and so on. Theoretical studies indicate that even in an ideal situation dynamical processes in DQD systems lead to asymmetry [9].

Recently, Fano resonance has attracted much research interest as an important interference effect in mesoscopic systems [10]. The effect has been studied theoretically for DQD systems with a special geometry [11] or for AharonovBohm interferometers with coupled QDs [12]. The interplay between interference and correlation effects has also been investigated and the coexistence of the Fano and Kondo resonances has been discussed [13, 14].

A double quantum dot can be regarded as an artificial molecule. In dependence on the strength of the inter-dot tunnel coupling, which can be easily tuned in experimental setup, QDs can form ionic-like (weak tunnel coupling) or covalent-like bonds (strong tunnel coupling). In the first case electrons are relatively well localized in individual quantum dots. When the tunnel coupling is strong, a DQD region can be treated as an artificial molecule with bonding and antibonding molecular states. Electrons can be regarded as delocalized over two quantum dots and transitions between molecular states rather than individual dot levels should be considered during transport processes. Two different mathematical approaches can be used to study electronic transport in these two regimes. When hopping between QDs is small in comparison to coupling with external electrodes $t<\Gamma$, it is quite natural to treat DQD and the leads as a one system and to apply an appropriate method to solve the problem. The basis of localized states can be used in this description and non-diagonal elements of density matrix should be taken into account [15]. On the other hand, in the regime of a strong inter-dot coupling $t \gg \Gamma$, from the physical point of view, it is more justified to treat the system as consisting of a molecule effectively coupled to reservoirs and to diagonalize the DQD Hamiltonian [16]. These two starting points were often used in theoretical studies of transport properties across coupled QDs (see e.g. [17-19]). Though the unitary transformation which diagonalizes the DQD Hamiltonian can be performed in equilibrium and non-equilibrium cases, both approaches are not fully equivalent as electrons at different states are spatially apart. It can influence the non-equilibrium Kondo effect in DQD systems as it was shown by Sun and Guo [20].

$I-V$ characteristics for molecules effectively coupled to external contacts were theoretically analyzed and mechanism of asymmetry observed in DQD systems was discussed [9]. It was shown that $I-V$ asymmetry in an ideal DQD system arises due to inelastic scattering between the states in the interacting region. Strong electron correlations play here an important role. Hamiltonian describing a DQD region was diagonalized and transitions between DQD molecular states during transport properties were investigated with the use of a diagrammatic technique for the Hubbard operator Green functions. Empty or singly occupied molecular states were considered in this approach. A role of many-particle states 
in coherent transport in a DQD system was studied in Refs. [21, 22] and contributions to transport from transmission channels through excited states were taken into account. Due to a complexity of the system with inter- and intra-dot correlations included, the DQD region was described in terms of its eigenstates given in atomic limit, namely disconnected of the leads. Such an approach can be only justified in a limit of a very strong coupling between quantum dots.

A renormalization of quantum dot levels due to tunnel coupling to reservoirs was included in approach proposed by Wunsch et al. and an influence of level shifts on $I-V$ characteristics was discussed [23]. It was shown that the renormalization of energy levels affects transport characteristics in a sequential limit leading to the negative differential conductance in the region of high bias voltages. The strong asymmetry in $I-V$ characteristics was also obtained.

In this paper a theoretical study of electron transport through a DQD system with the use of the non-equilibrium Green function formalism based on the equation of motion (EOM) method is presented. Two identical quantum dots, each with one energy level active in the transport, are coupled via tunneling processes with the hopping rate $t$ being of the same order as couplings with external contacts. Considering the intermediate regime with $t \approx \Gamma$ we treat a DQD region and external electrodes as a one system and solve an appropriate set of equations of motion. Inter-dot Coulomb interaction is taken into account and described by the Hubbard-like term with correlation parameter $U$. Calculations show that in DQD systems with energy level of quantum dots not aligned Coulomb correlations lead to more pronounced modifications than simple Coulomb blockade effects. These modifications are related to a strong dependence of population numbers on polarization of a bias voltage. For a one bias polarization below a certain voltage $\left|V_{0}\right|$ electrons are well localized in individual quantum dots with population close to $1 / 2$, whereas for the opposite polarization they start to be delocalized which influences transport properties considerably.

\section{Model}

A system under consideration consists of two QDs connected in series and coupled to external electrodes. It is described by the following Hamiltonian:

$$
H=H_{\mathrm{el}}+H_{\mathrm{DQD}}+H_{\mathrm{T}} .
$$

The first term in Eq. (1) is assumed in the form $H_{\mathrm{el}}=\sum_{k, \beta=\mathrm{L}, \mathrm{R}} \varepsilon_{k \beta} a_{k \beta}^{+} a_{k \beta}$ and describes left $(\beta=\mathrm{L})$ and right $(\beta=\mathrm{R})$ electrodes in the non-interacting quasiparticle approximation. $\varepsilon_{k \beta}$ is the single-electron energy in the electrode $\beta$ for the wave number $k$, whereas $a_{k \beta}^{+}$and $a_{k \beta}$ are the corresponding creation and annihilation operators. The term $H_{\mathrm{DQD}}$ describes the two-quantum-dot region and takes the form

$$
H_{\mathrm{DQD}}=\sum_{\beta=\mathrm{L}, \mathrm{R}}\left(E_{\beta} d_{\beta}^{+} d_{\beta}+t d_{\beta}^{+} d_{-\beta}+\frac{1}{2} U d_{\beta}^{+} d_{\beta} d_{-\beta}^{+} d_{-\beta}\right) .
$$


$E_{\beta}$ denotes here the energy of the discrete level and $d_{\beta}^{+}\left(d_{\beta}\right)$ is the creation (annihilation) operator of an electron in the quantum dot $\beta$. In Eq. (2) $-\beta$ corresponds to $\mathrm{R}$ for $\beta=\mathrm{L}$ and to $\mathrm{L}$ for $\beta=\mathrm{R}$. The second term describes the tunnel coupling between quantum dots and $t$ characterizes the tunnel strength. The inter-dot Coulomb interaction is taken into account via the Hubbard-like term with the correlation parameter $U$. We consider empty or singly occupied states on each QD and omit in the Hamiltonian terms describing the intra-dot Coulomb interactions. Tunnelling processes between the DQD region and external electrodes are described by the third part of the Hamiltonian (1) which is taken in the form

$$
H_{\mathrm{T}}=\sum_{k, \beta=\mathrm{L}, \mathrm{R}}\left(T_{k \beta} a_{k \beta}^{+} d_{\beta}+\text { h.c. }\right) \text {. }
$$

$T_{k \beta}$ are matrix elements describing electron tunnelling from the QD $\beta$ to the corresponding electrode.

\section{Non-equilibrium Green function formalism for DQD system}

Electric current flowing from the lead $\beta$ through the DQD region under a bias voltage applied is determined by the retarded (advanced) $G^{\mathrm{r}(\mathrm{a})}$ and correlation (lesser) $G^{<}$Green functions (GFs) and is given by the following formula [24]:

$$
I^{\beta}=\mathrm{i} \frac{2 e}{\hbar} \operatorname{Tr} \int \frac{\mathrm{d} E}{2 \pi} \hat{\Gamma}^{\beta}(E)\left\{\hat{G}^{<}(E)+f_{\beta}(E)\left[\hat{G}^{\mathrm{r}}(E)-\hat{G}^{\mathrm{a}}(E)\right]\right\},
$$

where $f_{\beta}(E)$ denotes the Fermi-Dirac distribution function for the $\beta$-th electrode and 2 is due to spin degeneracy. In the above expression the matrix $\hat{\Gamma}^{\beta}(E)$ with elements $\Gamma_{\beta_{1} \beta_{2}}^{\beta}(E)=2 \pi \sum_{k} T_{k \beta_{1}} T_{k \beta_{2}}^{*} \delta\left(E-\varepsilon_{k \beta}\right) \delta_{\beta \beta_{1}} \delta_{\beta_{1} \beta_{2}}$ describes the tunnelling coupling of the DQD region with $\beta$-th electrode. In the following we assume constant (independent of energy) coupling parameters $\Gamma_{\beta_{1} \beta_{2}}^{\beta}(E)=\Gamma_{\beta} \delta_{\beta_{1} \beta_{2}}$. Elements of the matrix $\hat{G}^{i}$ are defined in the following way: $G_{\beta \beta^{\prime}}^{i}(E)=\left\langle\left\langle d_{\beta}, d_{\beta^{\prime}}^{+}\right\rangle\right\rangle_{E}^{i}$ and correspond to the Fourier transforms of retarded, advanced and lesser Green functions for $i=\mathrm{r}, \mathrm{a},<$, respectively. The equation of motion method with the Hartree-Fock decoupling procedure is used to calculate retarded and advanced Green functions, whereas the correlation one is found according to a scheme proposed by Sun and Guo [20]. Function $G_{\beta \beta^{\prime}}(E)$ fulfils the following EOM:

$$
\left(E-E_{\beta}-\Sigma_{\beta}^{0}\right) G_{\beta \beta^{\prime}}=\delta_{\beta \beta^{\prime}}+U\left\langle\left\langle d_{\beta} d_{-\beta}^{+} d_{-\beta}, d_{\beta^{\prime}}^{+}\right\rangle\right\rangle+t G_{-\beta \beta^{\prime}},
$$

where $\Sigma_{\beta}^{0}$ corresponds to the self-energy of a non-interacting system. Higher order GFs $G_{\beta-\beta}^{2}=\left\langle\left\langle d_{\beta} d_{-\beta}^{+} d_{-\beta}, d_{\beta}^{+}\right\rangle\right\rangle$and $G_{-\beta \beta}^{2}=\left\langle\left\langle d_{-\beta} d_{\beta}^{+} d_{\beta}, d_{\beta}^{+}\right\rangle\right\rangle$appear in EOMs for $G_{\beta \beta}$ and $G_{-\beta \beta}$, respectively. Due to tunnelling processes between two quantum dots these functions are coupled in higher order equations and the appropriate set must be solved. No decoupling procedure is used for $G_{\beta-\beta}^{2}$ and $G_{-\beta \beta}^{2}$ functions. However, we decouple higher order functions containing $a_{k \beta}^{+}, a_{k \beta}$ operators apart from $d_{\beta}^{+}, d_{\beta}$ ones. The standard Hartree-Fock approximation is used which is fully justified for temperatures above the Kondo temperature. The solution of 
the obtained system of EOMs can be written in the compact matrix form which corresponds to the Dyson equation

$$
\hat{G}(E)=[\hat{I}-\hat{g}(E) \hat{\Sigma}(E)]^{-1} \hat{g}(E),
$$

where $\hat{g}(E)$ with elements $g_{\beta \beta^{\prime}}(E)=\delta_{\beta \beta^{\prime}}\left(E-E_{\beta}\right)^{-1}$ denotes the GF of two QDs in the absence of any coupling or interaction and the self-energy of the interacting system $\hat{\Sigma}$ takes the following form:

$$
\hat{\Sigma}(E)=\hat{g}^{-1}-\left[\hat{g}_{U}^{-1}-\hat{T}-U \hat{\tilde{n}}\right]^{-1}\left(\hat{g}_{U}^{-1}-\hat{T}\right)\left(\hat{g}^{-1}-\hat{T}\right)+\hat{\Sigma}^{0},
$$

where $g_{U \beta \beta^{\prime}}=\delta_{\beta \beta^{\prime}}\left(E-E_{\beta}-U\right)^{-1}, T_{\beta \beta^{\prime}}=\delta_{\beta-\beta^{\prime}} t$ and $\tilde{n}_{\beta \beta}=\left\langle d_{-\beta}^{+} d_{-\beta}\right\rangle$, $\tilde{n}_{\beta-\beta}=\left\langle d_{-\beta}^{+} d_{\beta}\right\rangle, \Sigma_{\beta \beta^{\prime}}^{0}=\Sigma_{\beta}^{0} \delta_{\beta \beta^{\prime}}$.

The correlation GF $G_{\beta \beta^{\prime}}^{<}$fulfils the following equation of motion [25]:

$$
G_{\beta \beta^{\prime}}^{<}=g_{\beta}^{<}\left\langle\left\{d_{\beta}, d_{\beta^{\prime}}^{+}\right\}\right\rangle+g_{\beta}^{\mathrm{r}}\left\langle\left\langle\left[d_{\beta}, H_{I}\right], d_{\beta^{\prime}}^{+}\right\rangle\right\rangle^{<}+g_{\beta}^{<}\left\langle\left\langle\left[d_{\beta}, H_{I}\right], d_{\beta^{\prime}}^{+}\right\rangle\right\rangle^{\mathrm{a}},
$$

where $g_{\beta}^{\mathrm{r}}(E)=\left(E-E_{\beta}+\mathrm{i} \eta\right)^{-1}, g_{\beta}^{<}(E)=2 \pi \mathrm{i} f_{\beta} \delta\left(E-E_{\beta}\right)$ are retarded and lesser functions of the uncoupled QD. All tunnelling and correlation effects are included in Hamiltonian $H_{I}$. The above formula can be also rewritten in the form

$$
g_{\beta}^{\mathrm{r}-1} G_{\beta \beta^{\prime}}^{<}=\left\langle\left\langle\left[d_{\beta}, H_{I}\right], d_{\beta^{\prime}}^{+}\right\rangle\right\rangle^{<}+g_{\beta}^{\mathrm{r}-1} g_{\beta}^{<}\left(E-E_{\beta}\right) G_{\beta \beta^{\prime}}^{\mathrm{a}},
$$

when the appropriate equation for the advanced GF is taken into account. Let us note that due to the presence of delta function $\delta\left(E-E_{\beta}\right)$ the last term in (9) will vanish after integration over energy. Applying the Langreth theorem to the lesser function $\left\langle\left\langle\left[d_{\beta}, H_{I}\right], d_{\beta^{\prime}}^{+}\right\rangle\right\rangle<[26]$ and adding the appropriate conjugated expression to formula (9) one can obtain the following set of equations when the integration over energy is taken [20]:

$$
\begin{gathered}
t\left(n_{\beta-\beta}-n_{-\beta \beta}\right)-\mathrm{i} \Gamma_{\beta} n_{\beta \beta}=\Gamma_{\beta} \int \frac{\mathrm{d} E}{2 \pi} f_{\beta}\left[G_{\beta \beta}^{\mathrm{r}}-G_{\beta \beta}^{\mathrm{a}}\right], \\
{\left[E_{-\beta}-E_{\beta}-\frac{\mathrm{i}}{2}\left(\Gamma_{\beta}+\Gamma_{-\beta}\right)\right] n_{\beta-\beta}+t\left(n_{\beta \beta}-n_{-\beta-\beta}\right)} \\
=\int \frac{\mathrm{d} E}{2 \pi}\left(\Gamma_{\beta} f_{\beta} G_{-\beta \beta}^{\mathrm{r}}-\Gamma_{-\beta} f_{-\beta} G_{-\beta \beta}^{\mathrm{a}}\right),
\end{gathered}
$$

where $n_{\beta \beta^{\prime}}=\left\langle d_{\beta}^{+} d_{\beta^{\prime}}\right\rangle=-\mathrm{i} \int \frac{\mathrm{d} E}{2 \pi} G_{\beta^{\prime} \beta}^{<}$. The procedure allows one to express the occupation numbers $n_{\beta} \equiv n_{\beta \beta}$ and correlation functions $n_{\beta \beta^{\prime}}$ in terms of retarded and advanced GFs $G_{\beta \beta^{\prime}}^{\mathrm{r}}, G_{\beta \beta^{\prime}}^{\mathrm{a}}$ and to omit explicit calculations of the lesser function $G_{\beta \beta^{\prime}}^{<}$. It can be applied if coupling parameters $\Gamma_{\beta}$ are independent of energy. In such a case electric current (Eq. (4)) and occupation numbers can be calculated self-consistently. A similar approach was used by Sun and Guo to investigate the Kondo resonance in DQD system [20].

\section{Results and discussion}

The symmetrical system with two identical QDs and with equal couplings to the leads is analyzed. Electric current flowing through the system in a non-equilibrium situation as well as the occupation numbers $n_{\beta}$ and correlation functions $n_{\beta-\beta}$ were calculated numerically in a self-consistent way with the use of 
the formalism described above. All parameters were measured in relative units with $\Gamma$ which represents the coupling strength to one of the electrodes taken as the energy unit. At first, we discuss the case when a hopping rate between quantum dots is equal to the tunnelling coupling $t=\Gamma_{\mathrm{L}}=\Gamma_{\mathrm{R}}=\Gamma$. We also assume $k T=\Gamma, E_{\mathrm{R}}=-4 \Gamma$ and treat the relative level spacing $\Delta E=E_{\mathrm{R}}-E_{\mathrm{L}}$ as a tuning parameter.

$I-V$ characteristics and differential conductance $G_{\text {diff }}=\mathrm{d} I / \mathrm{d} V$ calculated for various values of $\Delta E$ are presented in Fig. 1. To illustrate the influence of inter-dot correlation effects the results obtained for $U=0$ and $U=40 \Gamma$ are

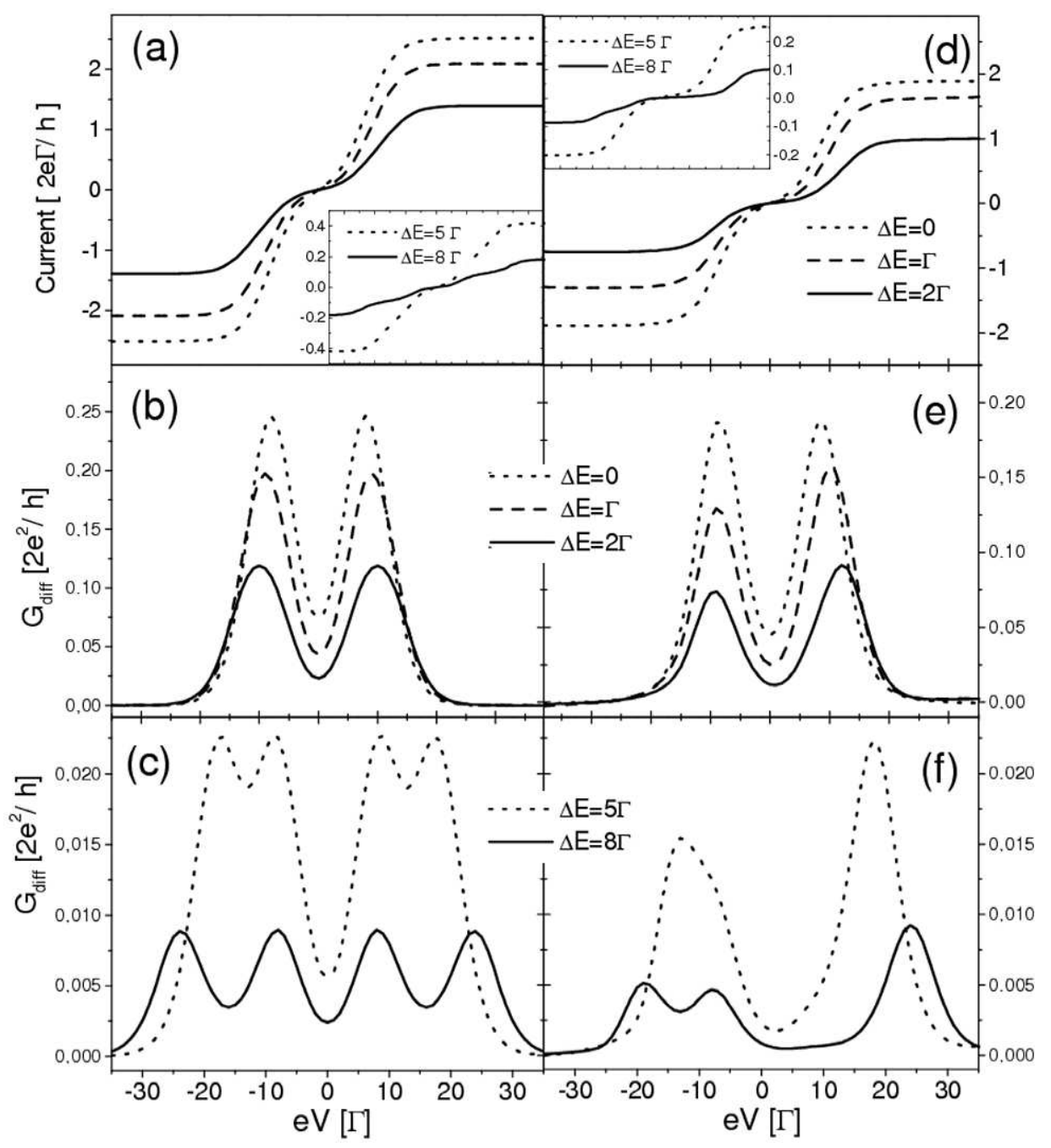

Fig. 1. Current-voltage (a, d) and differential conductance characteristics (b, c, e, f) calculated for indicated values of $\Delta E$ for uncorrelated (left part) and correlated systems (right part) with $U=0$ and $U=40 \Gamma$, respectively. 
depicted in the figure (left and right parts, respectively). Let us consider first the resonant case when positions of the discrete levels in two QDs are aligned and $\Delta E=0$. As expected, the current is fully symmetric with respect to the bias reversal both for $U=0$ and $U=40 \Gamma$. The symmetry can be well seen in Figs. 1b and e, where $G_{\text {diff }}$ is displayed. In the range of voltages presented in the figure the conductance shows two peaks, one for positive and one for negative voltages. Each of resonance peaks corresponds to transport through two molecular levels $E^{ \pm}=\frac{1}{2}\left(E_{\mathrm{L}}+E_{\mathrm{R}}\right) \pm \frac{1}{2} \sqrt{\Delta E^{2}+4 t^{2}}$ which cannot be resolved for $\Delta E=0$. It should be pointed out that shapes of these low-energy peaks in uncorrelated and correlated systems are similar and lower intensity of peaks presented by dotted line in Fig. 1e is the only sign of a presence of the Coulomb interaction. Therefore, in the resonance case with $\Delta E=0$ typical Coulomb blockade effects can be observed. With an increase in the level spacing the current strongly decreases, peaks in $G_{\text {diff }}$ start to shift and their intensities decrease. For higher values of $\Delta E$ conductance peaks split and molecular states $E^{+}, E^{-}$can be well resolved in characteristics of the non-interacting system (Fig. 1c). When correlation effects are included, intensities of the peaks which appear for positive and negative voltages are different and asymmetry takes place. As can be seen in Figs. 1e and $f$ the results strongly depend on the level off-set and the asymmetry is more pronounced for higher values of $\Delta E$. Let us note that for $\Delta E=8 \Gamma$ two well-defined peaks corresponding to molecular states $E^{+}$and $E^{-}$are visible for negative values of $e V$, whereas for opposite voltage polarization the states overlap giving one relatively high peak. Moreover, the transport is strongly blocked in a region of small positive values of $\mathrm{eV}$. The results clearly show that for DQD system with $\Delta E \neq 0$ modifications of conductance characteristics due to the inter-dot interaction are more pronounced than could be expected for simple Coulomb blockade effects.

The influence of the correlation parameter $U$ on electron transport is well illustrated in Fig. 2 in which the differential conductance is depicted for various values of $U$ and for $\Delta E=5 \Gamma$. Due to a large level separation $\Delta E$ two well-defined and symmetric peaks corresponding to molecular states $E^{+}$and $E^{-}$appear for negative and positive voltages when $U=0$ (thin solid line). With correlation effects included the peaks are shifted and asymmetry appears. For $U$ much larger than $\Delta E$ the changes are typical of systems with the Coulomb blockade. The conductance is suppressed in a low bias regime and it weakly depends on $U$. Positions of overlapping peaks related to molecular states $E^{+}$and $E^{-}$also weakly change with $U$. The corresponding Coulomb blockade peaks are shifted by $U$ and can be visible for $U=20 \Gamma$. Intensities of the peaks which appear for positive and negative voltages are different and asymmetry resulting from the presence of inter-dot interactions can be clearly seen. On the other hand, for $U$ close to $\Delta E$ transport through the system is considerably enhanced in a low energy region as a new channel opens when the level $E^{+}+U$ enters the bias window (Fig. 2, dashed lines). 


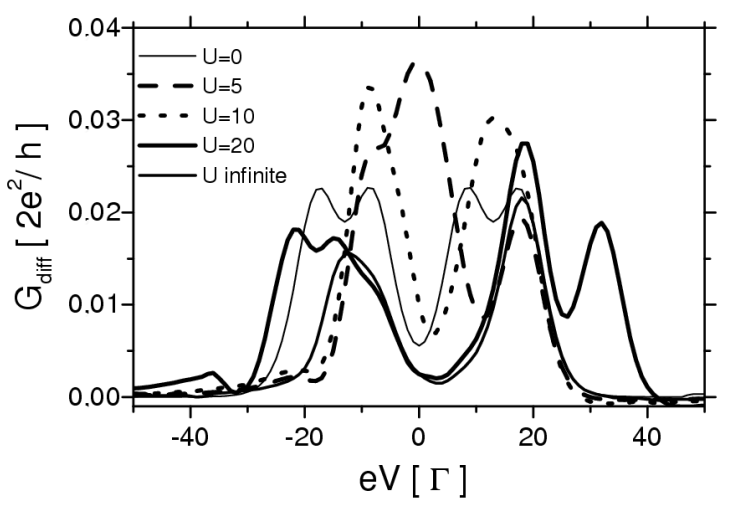

Fig. 2. Differential conductance for $\Delta E=5 \Gamma$ and indicated values of $U$.

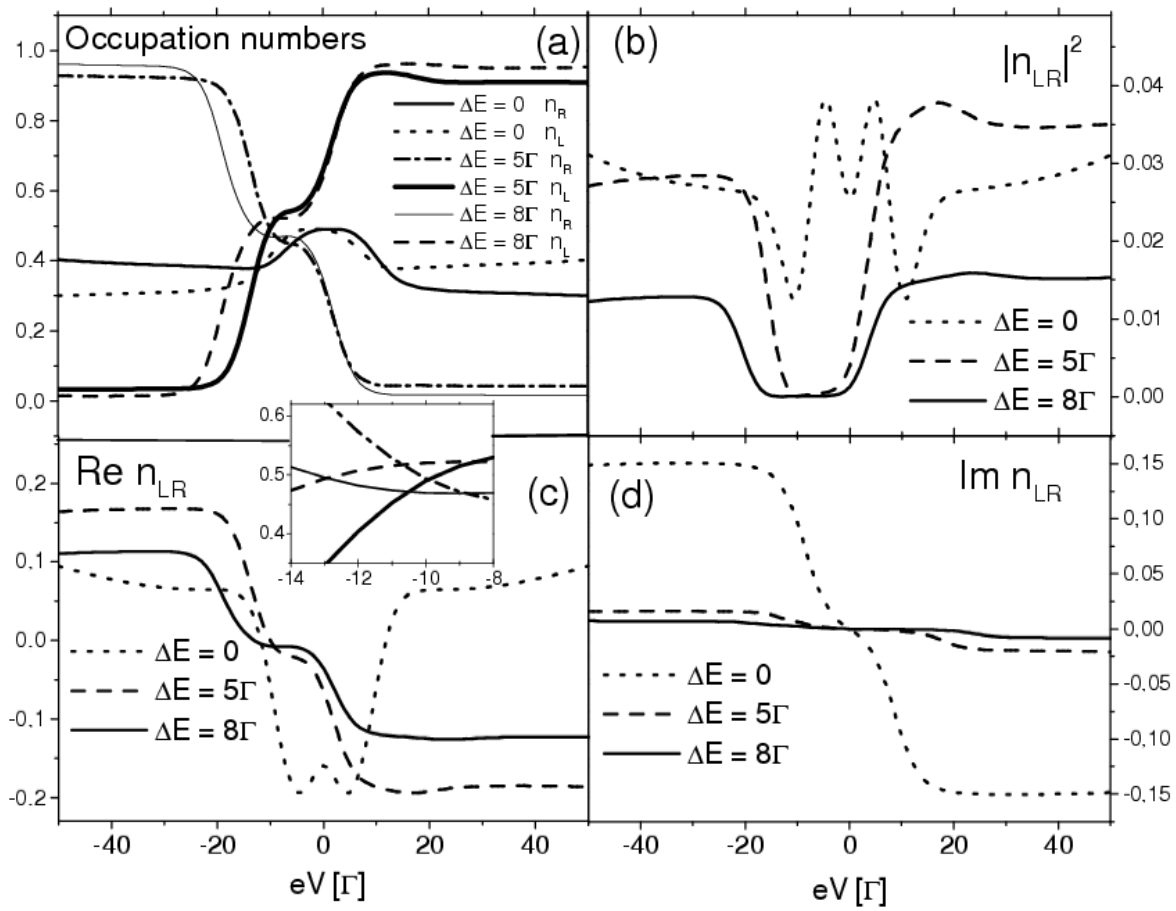

Fig. 3. Occupation numbers of left and right dots (a), probability $\left|n_{\mathrm{LR}}\right|^{2}$ (b), the correlation function $\operatorname{Re} n_{\mathrm{LR}}$ (c) and $\operatorname{Im} n_{\mathrm{LR}}$ (d) in dependence on $e V$ for $U=40 \Gamma$ and indicated values of $\Delta E$.

The occupation numbers of quantum dots $n_{\mathrm{L}}$ and $n_{\mathrm{R}}$ as well as the correlation function $n_{\mathrm{LR}}$ are presented in Fig. 3 for several values of $\Delta E$ and $U=40 \Gamma$. According to Fig. 3a one can see that for the case with level positions aligned $(\Delta E=0)$ the population numbers of both dots are close to $1 / 2$ in the vicinity of 
equilibrium. For higher values of $|e V|$ a mean number of electrons in each QD at first decreases and then remains almost constant. The probability $\left|n_{\mathrm{LR}}\right|^{2}$ shows oscillations in the low bias regime whereas for higher voltages it changes monotonically (Fig. 3b). When dot levels are not aligned $(\Delta E \neq 0)$ the population numbers of the left and right quantum dots are equal and close to $1 / 2$ for a certain negative (positive) value $e V_{0}$ when $\Delta E>0(\Delta E<0)$, as can be seen according to Fig. 3a. One can also observe that $\left|e V_{0}\right|$ increases with an increase in $\Delta E$ (the inset in Fig. 3). At higher voltages electrons start to accumulate on the QD close to the source electrode (the left one for $e V_{0}>0$ ), whereas the second QD becomes almost empty. It is interesting that for $\Delta E>0$ the function $\left|n_{\mathrm{LR}}\right|^{2}$ is practically equal to zero in a quite broad range of negative values of $e V$ (Fig. 3b), so electrons are well localized on the left and right quantum dots. However, for positive values of $e V$ the probability $\left|n_{\mathrm{LR}}\right|^{2}$ increases in a low bias regime and a pronounced asymmetry can be seen. Such behaviour strongly influences electric transport through the system.

Next, the influence of inter-dot coupling on the transport properties is investigated. As no special assumptions concerning $t$ have been introduced in EOM and higher order GFs have been split in a consistent way, the approach can be

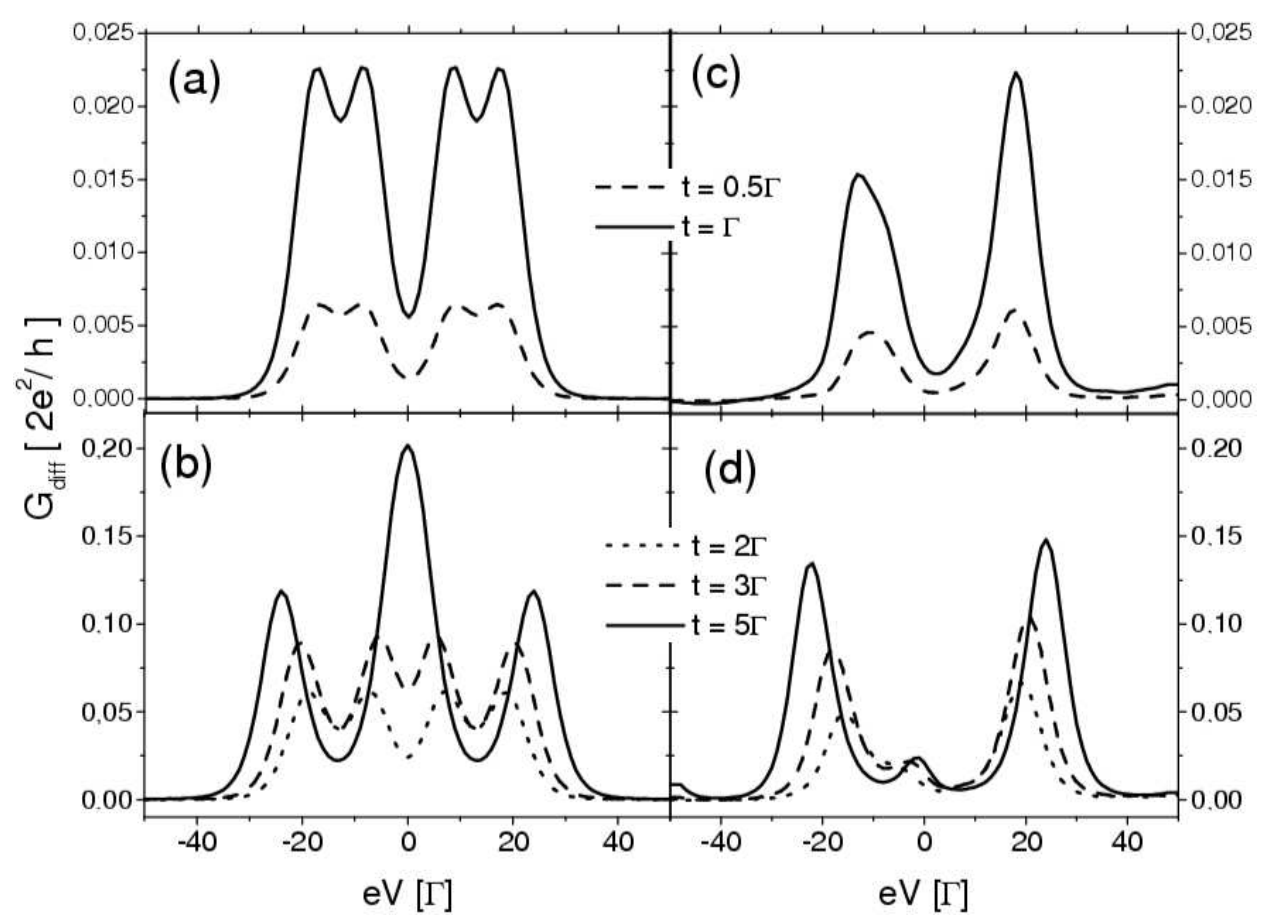

Fig. 4. Differential conductance of uncorrelated $(U=0$, left part) and correlated systems $(U=40 \Gamma$, right part) for indicated values of the inter-dot tunneling rate $t$ and $\Delta E=5 \Gamma$. 
used for a range of different hopping rates. Results obtained for the conductance in uncorrelated $(U=0$, left part) and correlated $(U=40 \Gamma$, right part) cases are depicted in Fig. 4 for several values of $t$. In uncorrelated system two molecular levels $E^{+}, E^{-}$are active in the transport both for positive and negative voltages. As $t$ increases the level separation $E^{+}-E^{-}$increases. The peaks shift apart and their intensities increase. For $t=5 \Gamma$ one of the levels is very close to the chemical potential of electrodes in equilibrium and the high peak builds up in the low voltage regime. Electron correlations strongly suppress the low voltage current and only a very low peak in $G_{\text {diff }}$ can be seen in this region (Fig. 4d). The influence of the Coulomb blockade for the case $t \leq \Gamma$ is much weaker.

In the limit of strong coupling between quantum dots $t \gg \Gamma$, two interacting quantum dots can be treated as a molecule weakly connected to external leads. In such a case mathematical description based on the diagonalization of the DQD Hamiltonian is more justified $[9,27]$. It is reasonable to compare the results of both approaches. Differential conductance as a function of bias voltage calculated for various values of the hopping parameter is depicted in Fig. 5. Two different methods are used, namely the formalism described in Sect. 3 (method 1) and the diagonalization procedure (method 2), which allows one to switch to a pseudo-spin representation [23]. Then the double QD problem shows a close analogy to

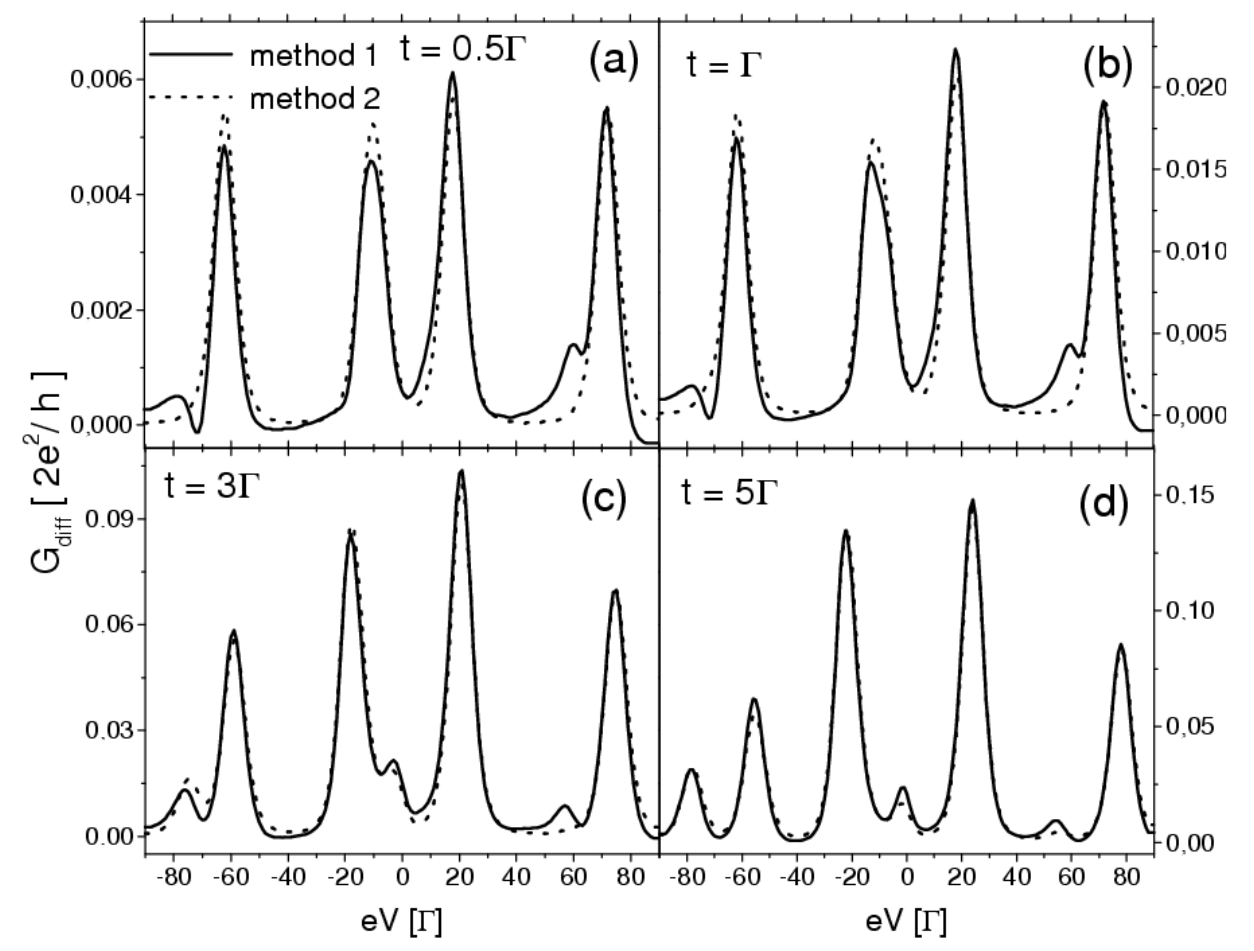

Fig. 5. Differential conductance calculated for indicated values of $t$ with the use of two approaches described in the text. $U=40 \Gamma$ and $\Delta E=5 \Gamma$. 
transport through a single magnetic quantum dot attached to ferromagnetic leads with antiparallel alignment of magnetic moments. According to Fig. 5 one can see that the results of both approaches are well consistent for high values of the hopping rate. When $t \leq \Gamma$ some discrepancies can be seen, though the positions of main peaks are well consistent (Figs. 5a and b). Let us note that the asymmetry shown by $G_{\text {diff }}$ is more pronounced when the presented formalism is used. This approach seems to be more justified from the physical point of view as weakly coupled quantum dots with $t \leq \Gamma$ can be hardly treated as a molecule.

\section{Summary and conclusions}

Effects of inter-dot correlations on electron transport across two QDs connected in series have been investigated. Results clearly show that correlations strongly modify $I-V$ characteristics. In a non-resonant case with energy levels of quantum dots not aligned conductance characteristics show asymmetry with respect to a bias voltage which considerably increases with an increase in the relative level spacing $\Delta E$. This conclusion is very well consistent with the results of theoretical studies performed by Fransson et al. [9] for similar systems. However, in contrast to the approach presented by Fransson our main approach is not based on the diagonalization procedure for $H_{\mathrm{DQD}}$ and it seems to constitute a more physical picture describing the transport across QDs with hopping rates comparable to couplings with external electrodes. Calculations show that the asymmetry in $I-V$ characteristics is related to the asymmetry in occupation numbers $n_{\mathrm{L}}$ and $n_{\mathrm{R}}$ of both quantum dots as well as in correlation function $\left|n_{\mathrm{LR}}\right|^{2}$. In a non-resonant case with $\Delta E \neq 0$ a certain voltage $V_{0}$ should be applied to equal population numbers in the left and right quantum dots. Moreover, for the one bias polarization electrons are well localized in the dots with probability $\left|n_{\mathrm{LR}}\right|^{2} \approx 0$, whereas for the opposite polarization some delocalization takes place. These processes strongly influence transport properties.

\section{References}

[1] T. Fujisawa, T. Hayashi, H.D. Cheong, Y.H. Jeong, Y. Hirayama, Physica E 21, 1046 (2004).

[2] V.N. Golovach, D. Loss, Semicond. Sci. Technol. 17, 355 (2002); D. Loss, D.P. DiVincenzo, Phys. Rev. B 57, 120 (1998).

[3] W.G. van der Wiel, S. De Franceschi, T. Fujisawa, J.M. Elzerman, S. Tarucha, L.P. Kouwenhoven, Rev. Mod. Phys. 75, 1 (2003).

[4] H. Jeong, A.M. Chang, M.R. Melloch, Science 293, 2221 (2001).

[5] R.H. Blick, A.K. Huttel, A.W. Holleitner, E.M. Hohberger, H. Qin, J. Kirschbaum, J. Weber, W. Wegscheider, M. Bichler, K. Eberl, J.P. Kotthaus, Physica E 16, 76 (2003).

[6] A.W. Holleitner, R.H. Blick, A.K. Huttel, K. Eberl, J.P. Kotthaus, Science 297, $70(2002)$. 
[7] T. Junno, S. Carlsson, H.Q. Xu, L. Samuelson, A.O. Orlov, G.L. Snider, Appl. Phys. Lett. 80, 667 (2002).

[8] K. Ishibashi, M. Suzuki, T. Ida, Y. Aoyagi, Appl. Phys. Lett. 79, 1864 (2001).

[9] J. Fransson, O. Eriksson, Phys. Rev. B 70, 085301 (2004); J. Fransson, Phys. Rev. B 69, 201304 (2004).

[10] I.G. Zacharia, D. Goldhaber-Gordon, G. Granger, M.A. Kastner, Yu.B. Khavin, H. Shtrikman, D. Mahalu, U. Meirav, Phys. Rev. B 64, 155311 (2001); J. Gores, D. Goldhaber-Gordon, S. Heemeyer, M.A. Kastner, H. Shtrikman, D. Mahalu, U. Meirav, Phys. Rev. B 62, 2188 (2000).

[11] M.L. Ladron de Guevara, F. Claro, P.A. Orellana, Phys. Rev. B 67, 195335 (2003).

[12] B. Kubala, J. Konig, Phys. Rev. B 65, 245301 (2002).

[13] B.R. Bułka, P. Stefański, Phys. Rev. Lett. 86, 5128 (2001).

[14] P. Stefański, A. Tagliacozzo, B.R. Bułka, Phys. Rev. Lett. 93, 186805 (2004).

[15] T.H. Stoof, Yu.V. Nazarov, Phys. Rev. B 63, 165313 (2001).

[16] G. Chen, G. Klimeck, S. Datta, G.H. Chen, W.A. Goddard, Phys. Rev. B 50, 8035 (1994); G. Klimeck, G.L. Chen, S. Datta, Phys. Rev. B 50, 2316 (1994).

[17] R. Ziegler, C. Bruder, H. Schoeller, Phys. Rev. B 62, 1961 (2000).

[18] P. Pals, A. MacKinnon, J. Phys., Condens. Matter 8, 5401 (1996).

[19] S. Lamba, S.K. Joshi, Phys. Rev. B 62, 1580 (2000).

[20] Q. Sun, H. Guo, Phys. Rev. B 66, 155308 (2002).

[21] B.R. Bułka, T. Kostyrko, Phys. Rev. B 70, 205333 (2004).

[22] J. Fransson, M. Rasander, Phys. Rev. B 73, 205333 (2006).

[23] B. Wunsch, M. Braun, J. König, D. Pfannkuche, Phys. Rev. B 72, 205319 (2005).

[24] Y. Meir, N.S. Wingreen, P.A. Lee, Phys. Rev. Lett. 66, 3048 (1991).

[25] C. Niu, D.L. Lin, T.H. Lin, J. Phys., Condens. Matter 11, 1511 (1999).

[26] H. Haug, A.P. Jauho, Quantum Kinetics in Transport and Optics of Semiconductors, Springer-Verlag, Berlin 1998.

[27] D. Sztenkiel, R. Świrkowicz, Acta Phys. Pol. A 110, 389 (2006). 